\title{
Natural Postoperative Bone Metabolic Changes after Total Knee Arthroplasty Determined by Positron Emission Tomography Scans
}

\author{
Masakazu Kanetaka', Soichiro Kaneko', Kei Wagatsuma ${ }^{2}$, Kenji Ishii ${ }^{2}$, Kimiteru Ito ${ }^{3}$, \\ Shinya Nakamura1, Hiroshi Hamaji' ${ }^{1}$, Yukimasa Yamato ${ }^{4}$, Yorito Anamizu${ }^{4}$, \\ Fumiaki Tokimura1, Tsuyoshi Miyazaki1 ${ }^{*}$
}

${ }^{1}$ Department of Orthopaedic Surgery, Tokyo Metropolitan Geriatric Hospital and Institute of Gerontology, Tokyo, Japan ${ }^{2}$ Research Team for Neuroimaging Research, Tokyo Metropolitan Geriatric Hospital and Institute of Gerontology, Tokyo, Japan ${ }^{3}$ Department of Diagnostic Radiology, Tokyo Metropolitan Geriatric Hospital and Institute of Gerontology, Tokyo, Japan ${ }^{4}$ Department of Spine Surgery, Tokyo Metropolitan Geriatric Hospital and Institute of Gerontology, Tokyo, Japan Email: *miyazak14@tmig.or.jp

How to cite this paper: Kanetaka, M., Kaneko, S., Wagatsuma, K., Ishii, K., Ito, K., Nakamura, S., Hamaji, H., Yamato, Y., Anamizu, Y., Tokimura, F. and Miyazaki, T. (2018) Natural Postoperative Bone Metabolic Changes after Total Knee Arthroplasty Determined by Positron Emission Tomography Scans. Open Journal of Orthopedics, 8, 443-457.

https://doi.org/10.4236/ojo.2018.812046

Received: August 30, 2018

Accepted: December 7, 2018

Published: December 10, 2018

Copyright (C) 2018 by authors and Scientific Research Publishing Inc. This work is licensed under the Creative Commons Attribution International License (CC BY 4.0).

http://creativecommons.org/licenses/by/4.0/

(c) (i) Open Access

\begin{abstract}
Artificial joint replacement surgery is the orthopedic procedure of choice to relieve pain, correct joint deformities, and help patients resume everyday activities. However, the detailed mechanisms regulating peri-implant bone remodeling at the bone-prosthesis interface remain elusive. To address this gap in knowledge, we evaluated the natural postoperative course of bone metabolism by using $\left[{ }^{18} \mathrm{~F}\right] \mathrm{NaF}$ positron emission tomography (PET)/computed tomography in 57 patients (104 joints) who underwent asymptomatic total knee arthroplasty (TKA). First, we measured total tracer uptake around TKA to determine the total bone metabolism (TBM) and used the ischial tuberosity as the reference tissue for normalization of PET images. Second, we calculated the TBM ratio (TBMR) by dividing the TBM by tracer uptake in the reference tissue. A moderate increase in TBMR was observed 4 - 5 days after implantation, and its intensity reached the maximum on the seventh postoperative day. The TBMR showed no differences until 12 weeks, after which it decreased slowly and returned to the basal levels. The new parameter TBMR and the unique pattern of postoperative metabolic changes in the bone around the prosthesis may help set accurate interpretation criteria to diagnose complications such as loosening or infections.
\end{abstract}

\section{Keywords}

NaF, PET, Bone Metabolism, Total Knee Arthroplasty, Ischial Tuberosity 


\section{Introduction}

Total joint arthroplasty is a surgical procedure in which parts of an arthritic or damaged joint are removed and replaced with a metal, plastic, or ceramic device called prosthesis. The prosthesis is designed to replicate the movement of a normal, healthy joint. Total knee arthroplasty (TKA) as well as total hip arthroplasty (THA) is the most commonly performed joint replacement procedures, although replacement surgery can be performed on other joints as well, including the shoulder, elbow, wrist, and ankle.

Knee replacement surgery was first performed in 1968, according to the American Academy of Orthopaedic Surgeons [1]. Since then, strong efforts have been made to improve the design and material properties of prostheses. In addition, advancements in surgical techniques, such as minimally invasive and muscle-sparing approaches, have greatly increased the effectiveness of this technique. A recent study reported that TKA is one of the most successful procedures in medical practice, and the literature contains a number of reports describing prosthesis survival rates of more than $90 \%$ at the 30 -year follow-up examination [2]. However, there is also a non-zero chance of recurring pain after TKA due to complications such as infections or loosening.

Nuclear medicine is a branch of medical imaging that uses small amounts of radioactive material to diagnose and determine the severity of or treat a variety of diseases, including many types of cancers, heart disease, gastrointestinal, endocrine, and neurological disorders, and other abnormalities within the body. Since nuclear medicine procedures can pinpoint molecular activity within the body, they offer the potential to identify disease in its earliest stages as well as show the patient's immediate response to therapeutic interventions.

$\left[{ }^{99 \mathrm{~m}} \mathrm{Tc}\right] \mathrm{MDP}$ bone scintigraphy is a frequently used radionuclide bone imaging technique, primarily due to its widespread availability. It can help diagnose a number of bone conditions, including metastasis of bone tumors, bone infection, and the location of bone inflammation and fractures. However, more recent reports have raised doubts over the previously reported effectiveness of bone scintigraphy, probably because its spatial resolution and sensitivity are lower than those of other diagnostic imaging techniques, including computed tomography (CT) and magnetic resonance imaging (MRI) [3].

Positron emission tomography (PET), which is noninvasive and usually painless with the exception of the intravenous injection, uses a positron emitter, a scintillator to detect annihilation photons, and a computer to help physicians diagnose and evaluate biochemical and physiological conditions. By providing molecular information, PET may identify body changes at the cellular level and detect the early onset of disease before it is evident on other imaging tests. At present, almost all PET scanners are combined with CT scanners to produce fused images and to perform various corrections using CT information. The combined PET/CT scans, therefore, provide images that pinpoint the anatomic location of abnormal metabolic activity within the body. These combined scans 
can provide more precise information and more accurate diagnoses than the two scans performed separately.

The recently developed ${ }^{18} \mathrm{~F}$-sodium fluoride $\left(\left[{ }^{18} \mathrm{~F}\right] \mathrm{NaF}\right)$ bone PET/CT is a more accurate diagnostic tool for specific bone disorders with the potential to replace conventional bone scintigraphy. Most of the $\mathrm{NaF}$ transported to the bone is retained only after a single pass of blood, making it a suitable radiopharmaceutical agent for the assessment of subtle changes in bone metabolic activity. By using a quantification parameter such as standardized uptake value (SUV), this method can yield more objective assessments than those obtained with conventional $\left[{ }^{99 \mathrm{~m}} \mathrm{Tc}\right]$ MDP studies. In addition, $\left[{ }^{18} \mathrm{~F}\right] \mathrm{NaF}$ PET/CT studies are more convenient for patients because the scanning time is less than that with conventional [ ${ }^{99 \mathrm{~m}} \mathrm{Tc}$ ] MDP bone scans.

In 2016, Son et al. [4] firstly reported the visual and quantitative analysis of $\left[{ }^{18} \mathrm{~F}\right] \mathrm{NaF}$ uptake after joint replacement. In their study, however, the interval between the surgery and the scan was from 3 to 25 months; $\left[{ }^{18} \mathrm{~F}\right] \mathrm{NaF}$ PET analysis was not performed within 3 months and after more than 25 months after the surgery. In this study, we investigated the tracer uptake not only within a week but also after more than 3 years after implantation to further investigate the utility of $\left[{ }^{18} \mathrm{~F}\right] \mathrm{NaF}$ PET/CT bone scans and the natural postoperative course in asymptomatic patients following joint replacement surgery.

\section{Materials and Methods}

\subsection{Patient Population}

From September 2014 to December 2017, a total of 57 patients (104 prostheses) without symptoms indicating post-TKA complications at the Tokyo Metropolitan Geriatric Hospital were enrolled in the current study, if they met all of the following inclusion criteria: 1) no subjective symptoms reported by the patient, 2) no radiolucent line on the postoperative plain radiograph, and 3) C-reactive protein within the normal ranges in the postoperative blood tests. This study was approved by the Institutional Review Board of Tokyo Metropolitan Geriatric Hospital and Institute of Gerontology, and all subjects gave their informed consent to participate in the study.

\section{2. [18F] NaF PET/CT Imaging}

$\left[{ }^{18} \mathrm{~F}\right] \mathrm{NaF}$ PET/CT images were obtained with a dedicated PET-CT scanner (Discovery PET/CT 710, GE Healthcare, Milwaukee, WI, USA) [5]. PET/CT imaging was performed $45 \mathrm{~min}$ after injection of $\left[{ }^{18} \mathrm{~F}\right] \mathrm{NaF}$ (3.7 MBq per kilogram of body weight). CT images at a rate of $3.27 \mathrm{~mm} /$ slice were acquired before PET acquisition for attenuation correction. PET data were acquired using a lutetium based scintillator with an axial field of view of $15.7 \mathrm{~cm}$. The spatial resolution determined in a performance test in NEMA NU 2-2007 was $4.52 \mathrm{~mm}$ at full width at half maximum (FWHM). The emission scan period per bed position was $1.5 \mathrm{~min}$; 13 or 14 bed positions were acquired. The average total PET/CT 
examination time was about $20 \mathrm{~min}$. All PET images were reconstructed under the following conditions: a three-dimensional-ordered-subsets expectation-maximization algorithm with time-of-flight and point-spread-functions, $192 \times 192$ matrix size, $3.65 \mathrm{~mm} / \mathrm{pixel}, 3.27 \mathrm{~mm} / \mathrm{slice}$, two iterations, 18 subsets, and a Gaussian filter of $4.0 \mathrm{~mm}$ (FWHM).

\section{3. [18F] NaF PET/CT Image Analysis}

First, the PET images were evaluated visually, after which the serial changes in tracer uptake were analyzed quantitatively based on the volume of interest (VOI) automatic isocontour method. All image processing procedures and measurements were performed using AW VolumeShare 7 (GE Healthcare, Milwaukee, WI, USA). For each image, the CT images were used for gross localization of bones and placement of the VOI for any given region. The average reference uptake value was calculated from two reference uptake values measured by two spherical VOIs placed at the ambilateral ischium. A three-dimensional cuboid around the prosthesis was systematically generated using AW VolumeShare 7 by specifying a length with the $x, y$, and $z$ axis directions. In this study, the total VOI was $13 \times 13 \times 18 \mathrm{~cm}$. Automatic isocontour detection was then used to refit the VOI around the prostheses by setting a threshold SUV of 4.0. For each contour, the $\mathrm{SUV}_{\text {mean }}, \mathrm{SUV}_{\text {max }}$, and $\mathrm{SUV}_{\text {peak }}$ were measured.

\subsection{Measurement of Metabolic Bone Volume and Total Bone Metabolism on [18F] NaF PET/CT}

Metabolic bone volume (MBV) was determined with a threshold SUV of 4.0. The contouring margins around the prostheses were automatically produced by the isocontour method. MBV was expressed as a volume $\left(\mathrm{cm}^{3}\right)$ containing an SUV intensity equal to or greater than 4.0. Total bone metabolism (TBM) was calculated by multiplying the $\mathrm{SUV}_{\text {mean }}$ value by the MBV. The TBM corresponds to the bone-forming activity in the entire area around the TKA.

\subsection{Statistical Analysis}

Inter-subject variability of $\mathrm{SUV}_{\text {Right-IschialTuberosity }}$ and $\mathrm{SUV}_{\text {Left-IschialTuberosity }}$ was analyzed in the whole patient group; for patients with two PET scans, only the first scan was used $(\mathrm{N}=69)$. Intra-subject variability of $\mathrm{SUV}_{\text {IschialTuberosity, which was }}$ the mean value of $S U V_{\text {Right-IschialTuberosity }}$ and SUV $V_{\text {Left-IschialTuberosity, was analyzed in a }}$ subgroup of 16 patients that underwent two scans on separate days. Inter- and intra-subject variabilities were assessed as standard deviation (SD) of the distribution of the respective SUV.

\section{Results}

\subsection{Patient Characteristics}

Seven $(12.3 \%)$ patients were men and fifty $(87.7 \%)$ were women; the average age 
of patients was $78.8 \pm 5.1$ years (range of $59-89 \mathrm{y}$ ). During the postoperative period (range of 4 - 4705 days), patients were evaluated during regular follow-ups and regarded as having no clinical evidence of complications.

\subsection{Visual Evaluation of [18F] NaF Distribution around TKA}

First, qualitative visual evaluation of tracer distribution was analyzed. One patient who had undergone left TKA four years previously was evaluated just before right TKA. As shown in Figure 1(a), mild homogeneous $\left[{ }^{18} \mathrm{~F}\right] \mathrm{NaF}$ uptake was observed in the left knee on the PET images. In contrast, the increased tracer accumulation in the right knee indicated bone and joint destruction related to the underlying osteoarthritis. Two weeks after right TKA, a second PET scan showed intense homogeneous uptake in all areas around the knee prosthesis, although $\mathrm{NaF}$ uptake around the left TKA was of very similar magnitude compared with that in the first scan (Figure 1(b)). To investigate the time course of bone metabolic activity after implantation of the prosthesis, PET scans of a 74-year-old woman with osteoarthritis of the right knee were obtained 5 and 23 days after right TKA. The uptake intensity on postoperative day (POD) 5 appeared to be lower than that on POD 23 (Figure 2), indicating ongoing up-regulation of bone-remodeling activity 5 days after surgery. Next, we analyzed PET images obtained from an 83-year-old woman who underwent left TKA and found that the uptake intensity on POD 7 appeared to be similar to that on POD 25 (Figure 3), suggesting that the uptake intensity reached the highest level 7 days after surgery and that the peak activity remained unchanged at least for approximately one month subsequently.

(a)

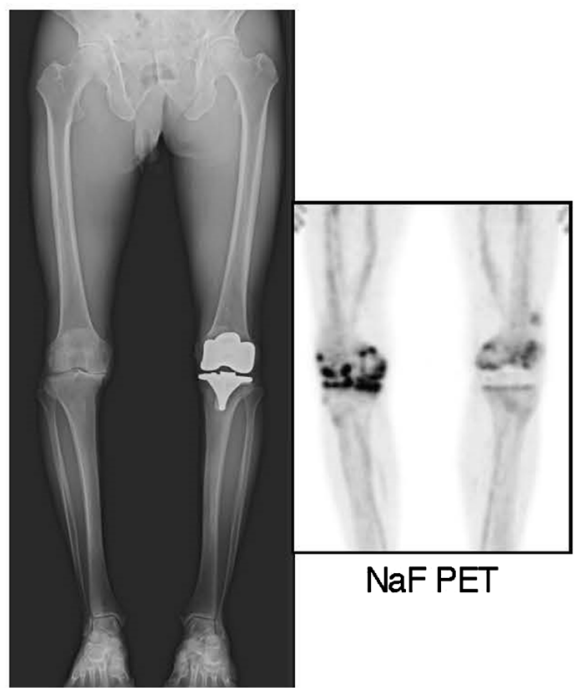

(b)

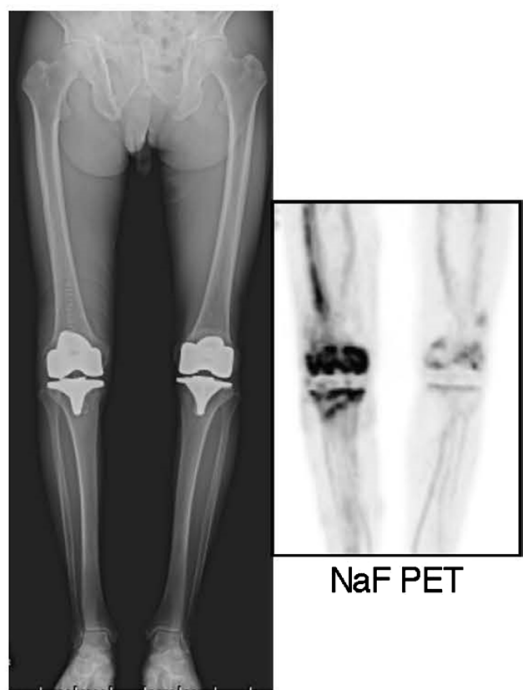

Figure 1. An 86-year-old man who had undergone left TKA 4 years ago without any complications received a right TKA for osteoarthritis of the knee. (a) NaF PET images obtained 2 weeks before right TKA showed almost normal accumulation around the left femoral and tibial components. (b) The repeat study performed 14 days after right TKA shows intensely increased radiopharmaceutical accumulation around the components. 
(a)

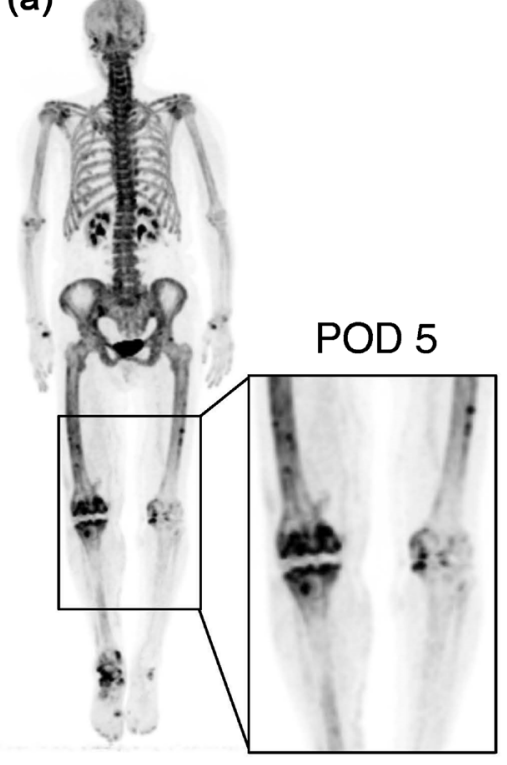

(c)

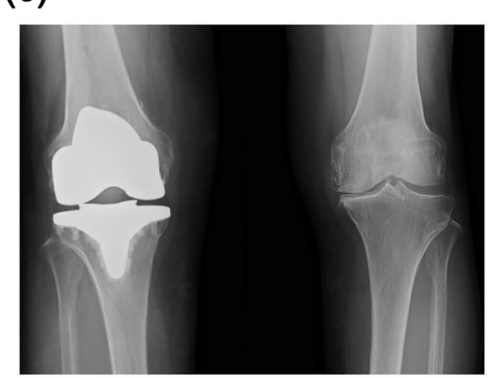

(b)

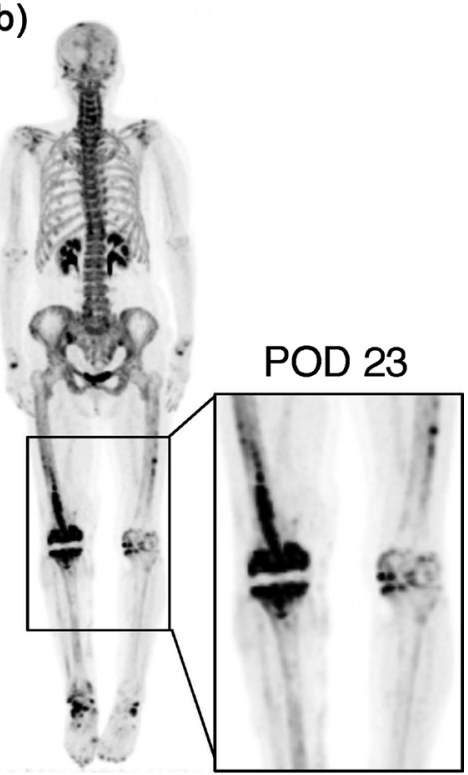

Figure 2. A 74-year-old woman received TKA for osteoarthritis of the right knee. Two NaF PET scans were performed on POD 5 (a) and POD 23 (b). The right knee showed less intense tracer uptake on POD 5 than on POD 23. (c) X-ray AP view of both knee joints after right TKA.

\subsection{Quantitative Evaluation of Tracer Distribution around TKA}

To evaluate the postoperative osteometabolic changes around the TKA, $\left[{ }^{18} \mathrm{~F}\right]$ $\mathrm{NaF}$ parameters such as the maximum standardized uptake value $\left(\mathrm{SUV}_{\max }\right)$, peak standardized uptake value $\left(\mathrm{SUV}_{\text {peak }}\right)$, and MBV were calculated. The $\mathrm{SUV}_{\max }$ is commonly used as a semiquantitative parameter in PET/CT studies and is valuable for diagnosis of various diseases and therapeutic responses. More recently, the $\mathrm{SUV}_{\text {peak }}$, which is defined as the mean tracer uptake within a spheric $1-\mathrm{cm}^{3}$ region around the area with the highest SUV, has been used to serve as a more reproducible parameter of maximum lesional uptake [6]. In addition, the MBV, obtained with a threshold SUV of 4.0 on 3D PET-CT images, is a possible indicator for response assessment of osteometabolic activity around TKA. Quantitative analysis of $\mathrm{SUV}_{\max }, \mathrm{SUV}_{\text {peak }}$, and $\mathrm{MBV}$ during the natural postoperative course showed that the $S U V_{\text {max }}, S U V_{\text {peak }}$, and MBV increased with time over the first 7 days after the arthroplasty, reaching the highest levels on POD 7 - 60 (7 - 60 days after implantation), and returned to the basal level after 1 year (Figure 4). 
(a)

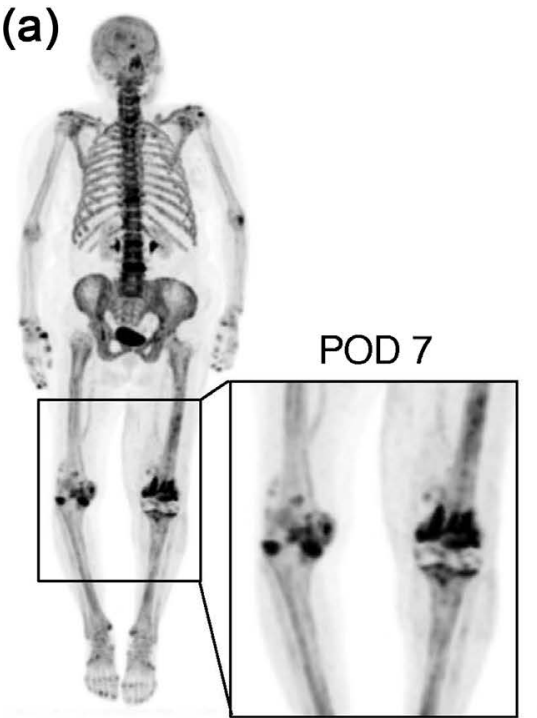

(b)

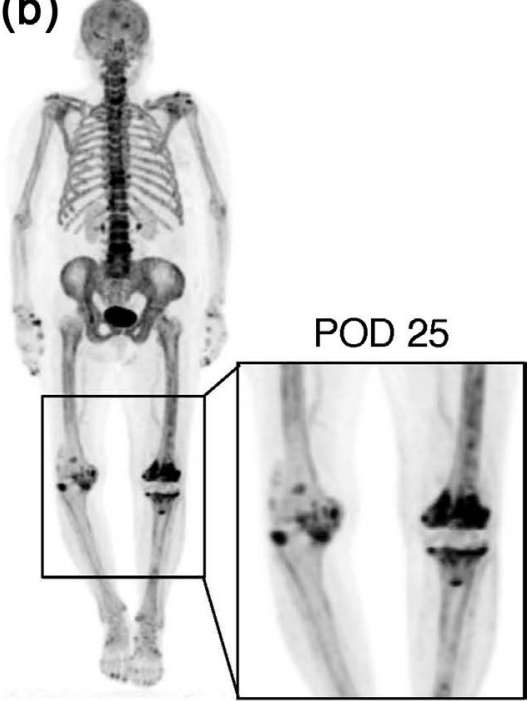

(c)

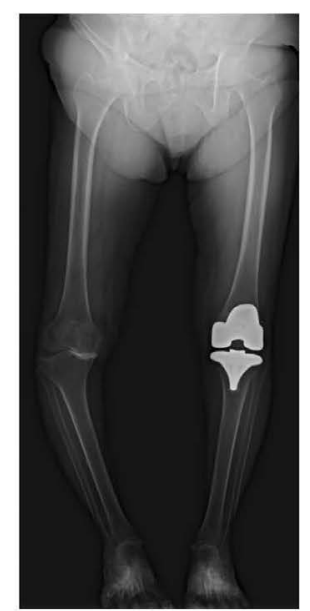

(d)

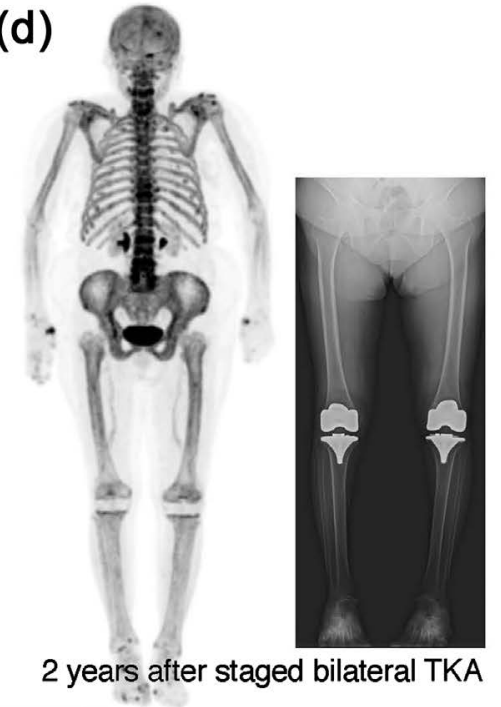

Figure 3. An 83-year-old woman received TKA for osteoarthritis of the left knee. Two NaF PET scans were performed on POD 7 (a) and POD 25 (b). There seemed to be no significant difference in NaF uptake between the two scans. (c) X-ray AP view of both knee joints in standing position just after left TKA. (d) Two years after staged bilateral TKA, both knees showed normal patterns of physiologic uptake of $\left[{ }^{18} \mathrm{~F}\right] \mathrm{NaF}$.

\subsection{Ischial Tuberosity as the Reference Region for Normalization of NaF PET Images}

To reduce inter- and intra-scanner differences in the NaF PET scans obtained from PET centers with or without the $\left[{ }^{18} \mathrm{~F}\right] \mathrm{NaF}$ synthesis facility, we hypothesized that normalization of tracer accumulation relative to a suitable reference tissue may improve accuracy. Uptake of $\left[{ }^{18} \mathrm{~F}\right] \mathrm{NaF}$ is not tumor-specific, and nonmalignant entities can also demonstrate subtle changes in radiotracer uptake in the bone and joint, including degenerative arthropathy, degenerative disk disease, facet arthropathy, rheumatoid arthritis, suspected stress fractures, early-stage rib fractures, bone graft viability, fracture nonunion, osteonecrosis of 


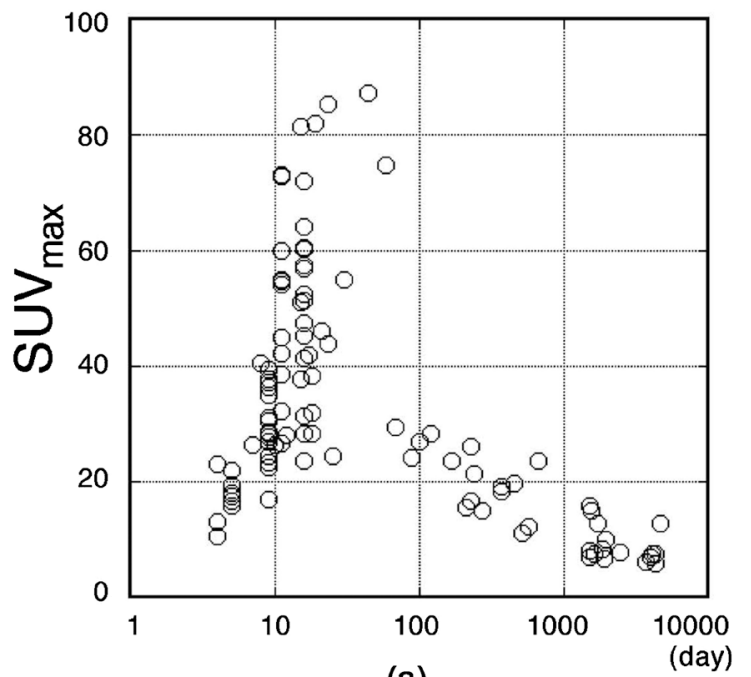

(a)

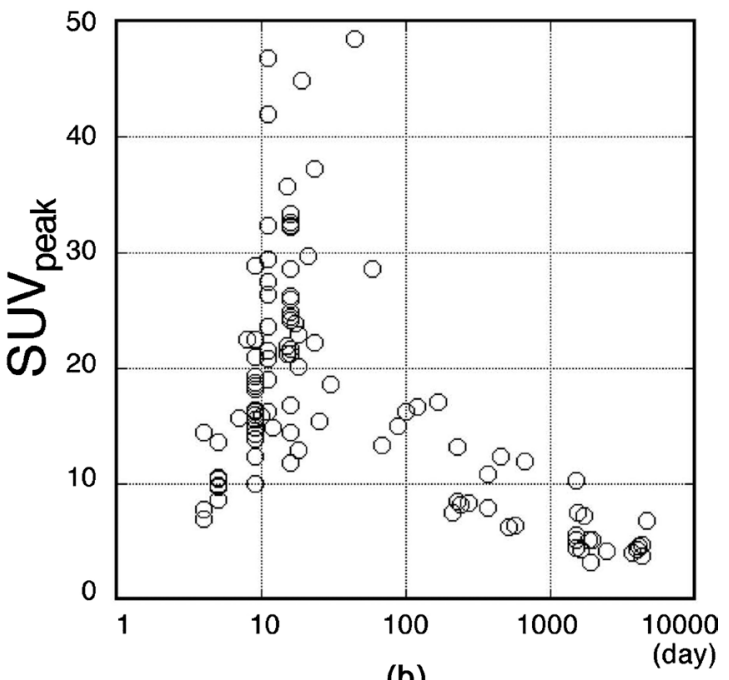

(b)

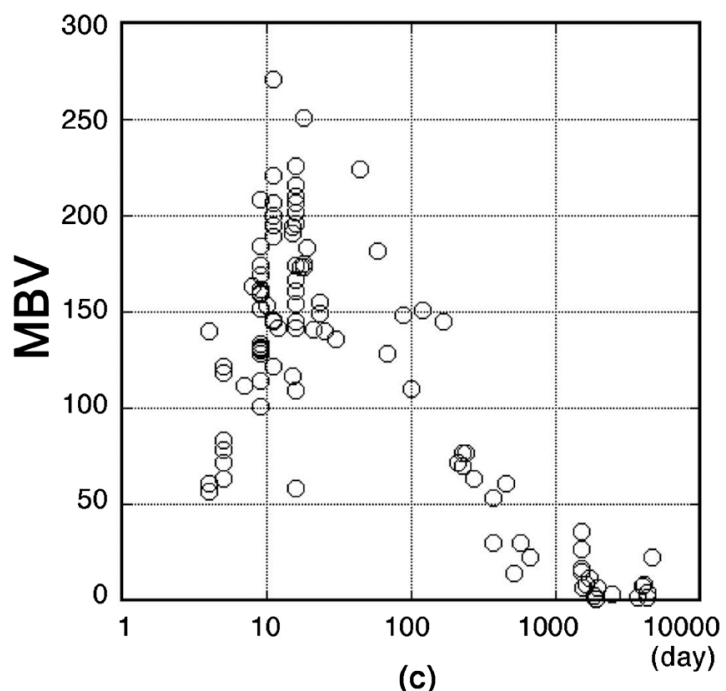

(c)

Figure 4. Time-activity curves of $\operatorname{SUV}_{\text {max }}$ (a) and $\mathrm{SUV}_{\text {peak }}$ (b) 30 min after intravenous injection of ${ }^{18} \mathrm{~F}-\mathrm{NaF}$ in patients. (c) Time-volume curves of metabolic bone volume (MBV) with the threshold SUV of 4.0 were expressed as volume $\left(\mathrm{cm}^{3}\right)$ containing SUV intensity of equal to or greater than 4.0.

the femoral head, and complications of joint prostheses. In this study, we focused on the ischial tuberosity as a reference region for normalization of PET activity (Figure 5(a)). The ischial tuberosity is a strong widening of the bone on either side of the frontal portion of the ischium, the lowest of the three major bones that make up each half of the pelvis. Since the ischial tuberosity is surrounded by soft tissues, including various muscles, and remains stable in comparison with the upper and lower extremities, degenerative changes in this region are relatively rare. To examine whether the ischial tuberosity is a suitable reference region, a sphere with a diameter of $1.5 \mathrm{~cm}$ was placed (drawn) in the right and left ischial tuberosities and the mean SUV in this region was quantitatively evaluated. The mean values of $\mathrm{SUV}_{\text {Right-IschalTuberosity }}$ and $\mathrm{SUV}_{\text {Left-IschialTuberosity }}$ across 73 investigated patients were $4.64 \pm 1.08$ and $4.61 \pm 1.01$, respectively. Quantitative evaluation revealed a high correlation between the mean SUVs 

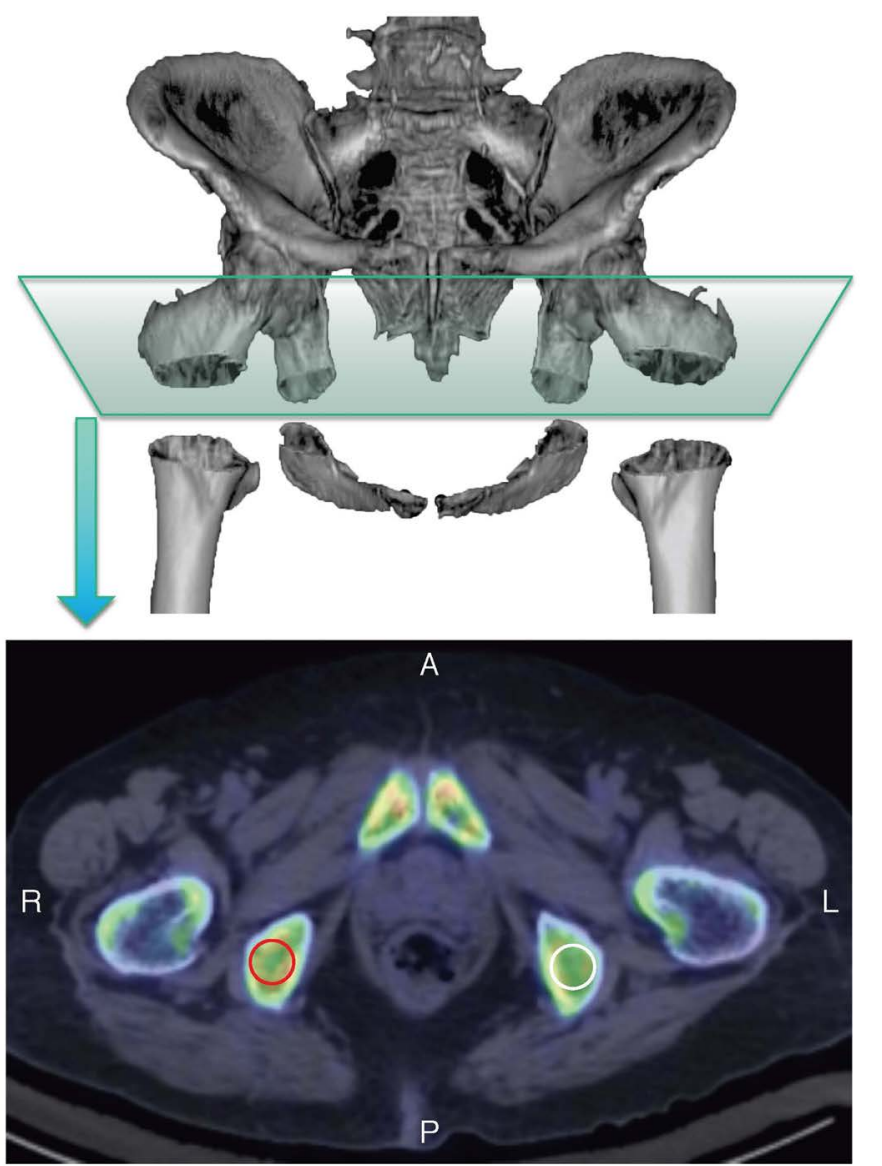

(a)

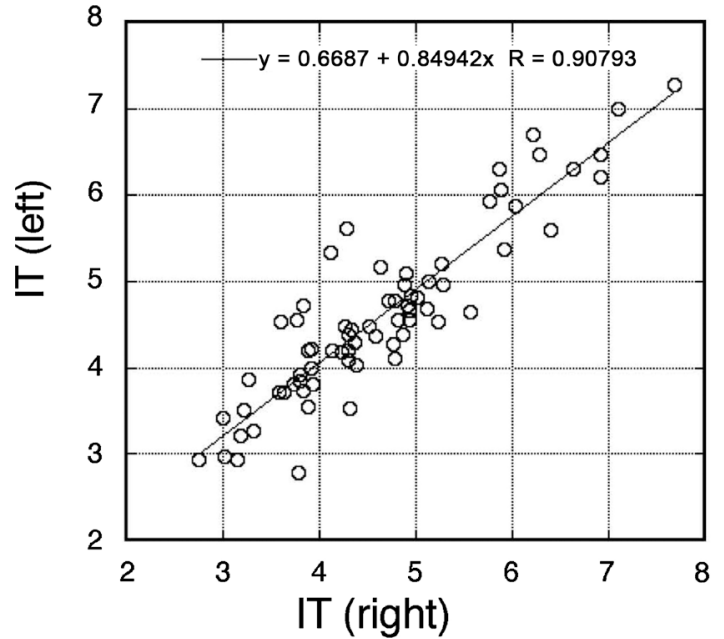

(b)

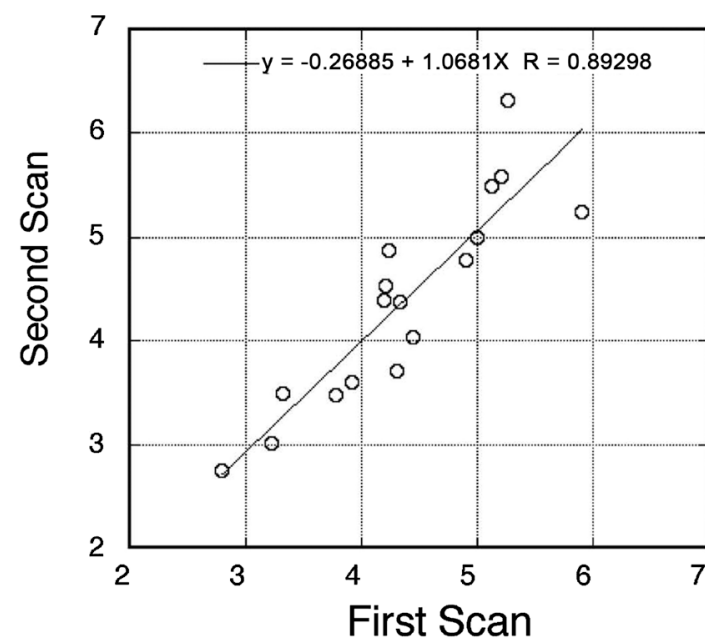

(c)

Figure 5. Ischial tuberosity as the reference region for normalization of NaF uptake. (a) An example of VOI within the right and left ischial tuberosities is displayed in the 3DCT image of the pelvis. The lower panel shows an axial fused NaF PET/CT image with two spheres of $1.5-\mathrm{cm}$ diameter drawn in the right (red circle) and left (white circle) ischial tuberosities. $\mathrm{L}=$ left, $\mathrm{R}=$ right, $\mathrm{A}=$ anterior, $\mathrm{P}=$ posterior; (b) Correlation ana-

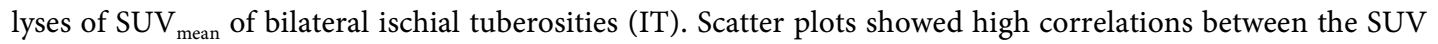
values of the right and left ischial tuberosities, $\mathrm{p}=0.567973452$; (c) Correlation between the first and second scans of $S_{U V} V_{\text {IscicalTuberosity. }}$ Scatter plots showed good test-retest reliability, $\mathrm{p}=0.796451829$. 
obtained from the ischial tuberosities on both sides $(\mathrm{R}=0.90793, \mathrm{p}=$ 2.28755E-15) (Figure 5(b)). As shown in Figure 5(c), correlation analysis revealed a linear correlation between the first and second scans in 16 patients receiving two PET scans on different days. The mean values of SUV $\mathrm{IschialTuberosity}_{\text {in }}$ the first and second scans were $4.36 \pm 0.81$ and $4.39 \pm 0.97$, respectively $(\mathrm{R}=$ $0.89298, \mathrm{p}=3.23608 \mathrm{E}-6)$.

\subsection{Measurement of Total NaF Accumulation around TKA Normalized to the Reference Tissue}

Semiquantitative parameters such as the standardized uptake value ratio (SUVR) require normalization of the radiotracer activity to a reference tissue to allow monitoring of changes in tracer accumulation [7]. Here, we measured total tracer uptake around TKA as TBM, which is calculated by multiplying the SUV $\mathrm{V}_{\text {mean }}$ by the $\mathrm{MBV}_{4.0}$. Furthermore, we also determined the TBM ratio (TBMR), which is normalized by dividing the TBM by tracer uptake in reference tissue. As shown in Figure 6, TBMR showed a time-dependent increase for the first 7 days after implantation and then remained at the highest level throughout the first 2 months. Thereafter, it gradually declined to the basal levels.

\section{Discussion}

In this study, we performed both visual and quantitative evaluation of the bone metabolic activity and investigated natural post-operative serial changes in uptake intensity $\left(\mathrm{SUV}_{\max }, \mathrm{SUV}_{\text {peak }}, \mathrm{MBV}, \mathrm{TBM}\right.$, and $\left.\mathrm{TBMR}\right)$ during the period spanning from within a week to more than 3 years after surgery.

To date, ${ }^{99 \mathrm{~m}} \mathrm{Tc}$ has been the standard bone imaging radiopharmaceutical agent for bone scintigraphy, which has been utilized for oncological evaluations and has been considered to be a routine procedure for the assessment of bone involvement for many decades. The ${ }^{18} \mathrm{~F}$-fluoride ion $\left(\left[{ }^{18} \mathrm{~F}\right] \mathrm{NaF}\right)$ was introduced as
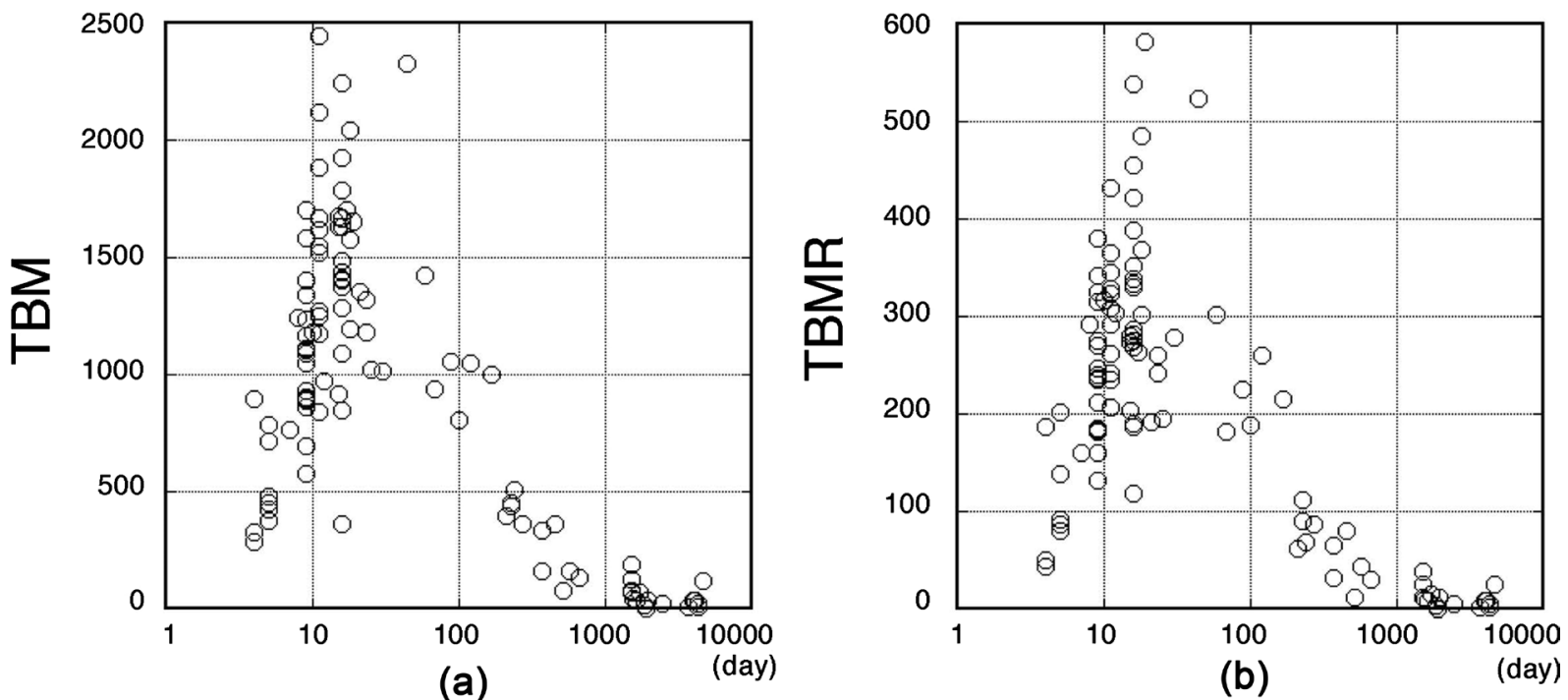

Figure 6. Changes in TBM or TBMR during the natural postoperative course of knee prostheses. 
a bone-seeking agent by Blau et al. in 1962 [8] and approved by the FDA for detection of osteogenic activity in 1972. Unfortunately, it lost popularity due to the limited availability of cyclotron generators and its short half-life. In the 1990s, PET technology spread worldwide, and $\left[{ }^{18} \mathrm{~F}\right] \mathrm{NaF}$ PET regained interest as a more accurate diagnostic tool for specific bone disorders. With the appearance of multi-slice PET devices with high resolution and high sensitivity, systemic three-dimensional tomograms have become easily obtainable, making it possible to use bone PET to rapidly search for systemic bony lesions (Supplementary Figure S1). In addition, $\left[{ }^{18} \mathrm{~F}\right] \mathrm{NaF}$ PET/CT studies are more convenient for patients because the scanning time is less than that with conventional [ ${ }^{99 \mathrm{~m}} \mathrm{Tc}$ ] MDP studies. Furthermore, NaF PET scans are also useful for assessing subtle changes in three-dimensional volumetric structures and for measurement of quantitative parameters. Hence, we believe that $\left[{ }^{18} \mathrm{~F}\right] \mathrm{NaF}$ PET would eventually replace conventional bone scintigraphy for the assessment of bone and joint abnormalities where PET scanners are available.

$\mathrm{SUV}_{\max }$ is a widely used quantitative parameter in ${ }^{18} \mathrm{~F}$-FDG PET, and other volume-based PET parameters such as the metabolic tumor volume (MTV) and total lesion glycolysis (TLG) are dependent on $\mathrm{SUV}_{\max }$ [9]. These PET parameters have limited effectiveness in terms of a straightforward assessment of metabolic characteristics, because various factors such as body weight, obesity, blood glucose level, and post-injection uptake time can affect the $\mathrm{SUV}_{\max }$ [10]. In addition, several studies have reported that the liver is widely used as a reference region [11] and that the tumor-to-liver ratio of FDG uptake is useful for predicting treatment responses in malignant patients [12] [13] [14]. In this context, we selected the ischial tuberosity as the reference region for normalization of $\mathrm{NaF}$ PET images, and we believe that the ischial tuberosity is an ideal reference region for bone PET scans (Figure 5).

The uptake patterns found in our study show some differences from those found in previous studies [4]. Our study describes a gradual increase in bone-forming activity for the first 7 days after surgery. This bone metabolic response to implantation indicates the existence of an unknown mechanism that regulates the time-dependent biological changes at the bone-prosthesis interface. Since bone cement [15] was used in all knee arthroplasties in our study, one possible explanation for this response pattern is that cell death at the bone-cement interface may be induced by hyperthermia immediately after fixation of all the components. After thermal injury to bone cells, the subsequent recruitment of osteoblastic cells around the cement layer may play an important role in repair of the damaged bone, leading to the higher bone-forming activity shown in Figures 2-4, and Figure 6.

During TKA, the femoral canal was opened with an intramedullary drill for insertion of the femoral intramedullary guiding rod for appropriate femoral component positioning. As shown in Figure 2, the time course of bone metabolic activity in the distal half of the right femur was similar to that of the 
bone-cement interface around the TKA prosthesis, although no implant and cement were placed in the distal femoral medullary cavity. These results suggest that thermal injury in bone cells is not involved in the typical time course of bone metabolic activity around the knee prostheses.

Another possible hypothesis is that the mechanical loading in weight-bearing activities may be involved in bone metabolic activity around implants following surgery. Interestingly, we found a higher intensity of radiotracer uptake at the distal area and the posterior condyle area compared with that at the anterior flange area as a typical uptake pattern around the femoral components (data not shown). In general, mechanical loading in the knee, especially at the distal surface of the femur and posterior condyle area, is much higher than that at the patellar surface of the femur during three common daily activities: walking, ascending stairs, and rising from a chair. There is a possibility that the gradual up-regulation of bone-forming activity for the first 7 days after implantation might be due to earlier and more intensive physiotherapy exercise with full-weight-bearing following surgery. The time course of bone metabolic activity around shoulder prostheses, however, was similar to that of the bone-cement interface around TKA prostheses (data not shown), although the mechanical stress of the nonweight-bearing upper extremity is supposed to be less than that of the weight-bearing lower extremity. Those results suggest that mechanical stress does not appear to be involved in the typical time course of bone metabolic activity around knee prostheses.

Limitations to the widespread use of PET arise from the high costs of cyclotrons needed to produce the short-lived radionuclides for PET scanning and the need for specially adapted on-site chemical synthesis apparatus to produce the radiopharmaceuticals after radioisotope preparation. Thus, it is important for the patient to be on time for the appointment and to receive the radioactive material at the scheduled time. Late arrival for an appointment may require rescheduling the procedure for another day. These limitations should be overcome in the future.

\section{Conclusion}

Using NaF PET scans for the evaluation of peri-implant bone remodeling at the interface, we revealed the natural postoperative bone metabolic changes with time after TKA in asymptomatic patients. After arthroplasty, bone metabolic activity increased with time over the first 7 days, reaching the highest level on POD 7 - 60, and then returned to the basal level thereafter. Another clinical significance of our work is that this is the first study providing information concerning the usefulness of the ischial tuberosity as a reference tissue. Furthermore, this study has shown that volume-based PET functional parameters such as TBM and TBMR can provide metabolic information regarding artificial joint prostheses after surgery. The new parameter TBMR could have significant value for the detection and diagnosis of delayed complications, including loosening or infec- 
tions, although multicenter studies are required to clarify whether it is useful to reduce inter-scanner variability. Further investigation of the serial physiological changes of bone turnover with time after TKA in patients will provide new insights into the molecular mechanisms regulating bone metabolism at the bone-prosthesis interface.

\section{Acknowledgements}

This paper was supported by Grant-in-Aid for Translational Research, Tokyo, Metropolitan Institute of Gerontology, scholarship grant from Pfizer Health Research Foundation, and scholarship grant from TEIJIN PHARMA LIMITED.

\section{Conflicts of Interest}

The authors declare no conflicts of interest regarding the publication of this paper.

\section{References}

[1] TREATMENT Total Knee Replacement. https://orthoinfo.aaos.org/en/treatment/total-knee-replacement/

[2] Ritter, M.A., Keating, E.M., Sueyoshi, T., Davis, K.E., Barrington, J.W. and Emerson, R.H. (2016) Twenty-Five-Years and Greater, Results after Nonmodular Cemented Total Knee Arthroplasty. Journal of Arthroplasty, 31, 2199-2202. https://doi.org/10.1016/j.arth.2016.01.043

[3] O’Sullivan, G.J., Carty, F.L. and Cronin, C.G. (2015) Imaging of Bone Metastasis: An Update. World Journal of Radiology, 7, 202-211. https://doi.org/10.4329/wjr.v7.i8.202

[4] Son, H.J., Jeong, Y.J., Yoon, H.J., Wang, L., Kim, H.J., Park, J.H. and Kang, D.Y. (2016) Visual Pattern and Serial Quantitation of (18)F-Sodium Fluoride PET/CT in Asymptomatic Patients after Hip and Knee Arthroplasty. Nuclear Medicine and Molecular Imaging, 50, 308-321. https://doi.org/10.1007/s13139-016-0430-0

[5] Bettinardi, V., Presotto, L., Rapisarda, E., Picchio, M., Gianolli, L. and Gilardi, M.C. (2011) Physical Performance of the New Hybrid PETCT Discovery-690. Medical Physics, 38, 5394-5411. https://doi.org/10.1118/1.3635220

[6] Wahl, R.L., Jacene, H., Kasamon, Y. and Lodge, M.A. (2009) From RECIST to PERCIST: Evolving Considerations for PET Response Criteria in Solid Tumors. The Journal of Nuclear Medicine, 50, 122S-150S. https://doi.org/10.2967/jnumed.108.057307

[7] Shokouhi, S., McKay, J.W., Baker, S.L., Kang, H., Brill, A.B., Gwirtsman, H.E., Riddle, W.R., Claassen, D.O. and Rogers, B.P. (2016) Reference Tissue Normalization in Longitudinal (18)F-Florbetapir Positron Emission Tomography of Late Mild Cognitive Impairment. Alzheimer's Research \& Therapy, 8, 2. https://doi.org/10.1186/s13195-016-0172-3

[8] Blau, M., Nagler, W. and Bender, M.A. (1962) Fluorine-18: A New Isotope for Bone Scanning. The Journal of Nuclear Medicine, 3, 332-334.

[9] Moon, S.H., Hyun, S.H. and Choi, J.Y. (2013) Prognostic Significance of Volume-Based PET Parameters in Cancer Patients. Korean Journal of Radiology, 14, 1-12. https://doi.org/10.3348/kjr.2013.14.1.1 
[10] Adams, M.C., Turkington, T.G., Wilson, J.M. and Wong, T.Z. (2010) A Systematic Review of the Factors Affecting Accuracy of SUV Measurements. American Journal of Roentgenology, 195, 310-320. https://doi.org/10.2214/AJR.10.4923

[11] Kamibayashi, T., Tsuchida, T., Demura, Y., Tsujikawa, T., Okazawa, H., Kudoh, T. and Kimura, H. (2008) Reproducibility of Semi-Quantitative Parameters in FDG-PET Using Two Different PET Scanners: Influence of Attenuation Correction Method and Examination Interval. Molecular Imaging and Biology, 10, 162-166. https://doi.org/10.1007/s11307-008-0132-9

[12] Findlay, M., Young, H., Cunningham, D., Iveson, A., Cronin, B., Hickish, T., Pratt, B., Husband, J., Flower, M. and Ott, R. (1996) Noninvasive Monitoring of Tumor Metabolism Using Fluorodeoxyglucose and Positron Emission Tomography in Colorectal Cancer Liver Metastases: Correlation with Tumor Response to Fluorouracil. Journal of Clinical Oncology, 14, 700-708. https://doi.org/10.1200/JCO.1996.14.3.700

[13] Flamen, P., Van Cutsem, E., Lerut, A., Cambier, J.P., Haustermans, K., Bormans, G., De Leyn, P., Van Raemdonck, D., De Wever, W., Ectors, N., Maes, A. and Mortelmans, L. (2002) Positron Emission Tomography for Assessment of the Response to Induction Radiochemotherapy in Locally Advanced Oesophageal Cancer. Annals of Oncology, 13, 361-368. https://doi.org/10.1093/annonc/mdf081

[14] Song, M.J., Bae, S.H., Yoo Ie, R., Park, C.H., Jang, J.W., Chun, H.J., Choi, B.G., Lee, H.G., Choi, J.Y. and Yoon, S.K. (2012) Predictive value of (1)(8)F-Fluorodeoxyglucose PET/CT for Transarterial Chemolipiodolization of Hepatocellular Carcinoma. World Journal of Gastroenterology, 18, 3215-3222.

[15] O’Rourke, M.R., Callaghan, J.J., Goetz, D.D., Sullivan, P.M. and Johnston, R.C. (2002) Osteolysis Associated with a Cemented Modular Posterior-Cruciate-Substituting Total Knee Design: Five to Eight-Year Follow-Up. The Journal of Bone and Joint Surgery, 84, 1362-1371. https://doi.org/10.2106/00004623-200208000-00012 


\section{Supplementary}
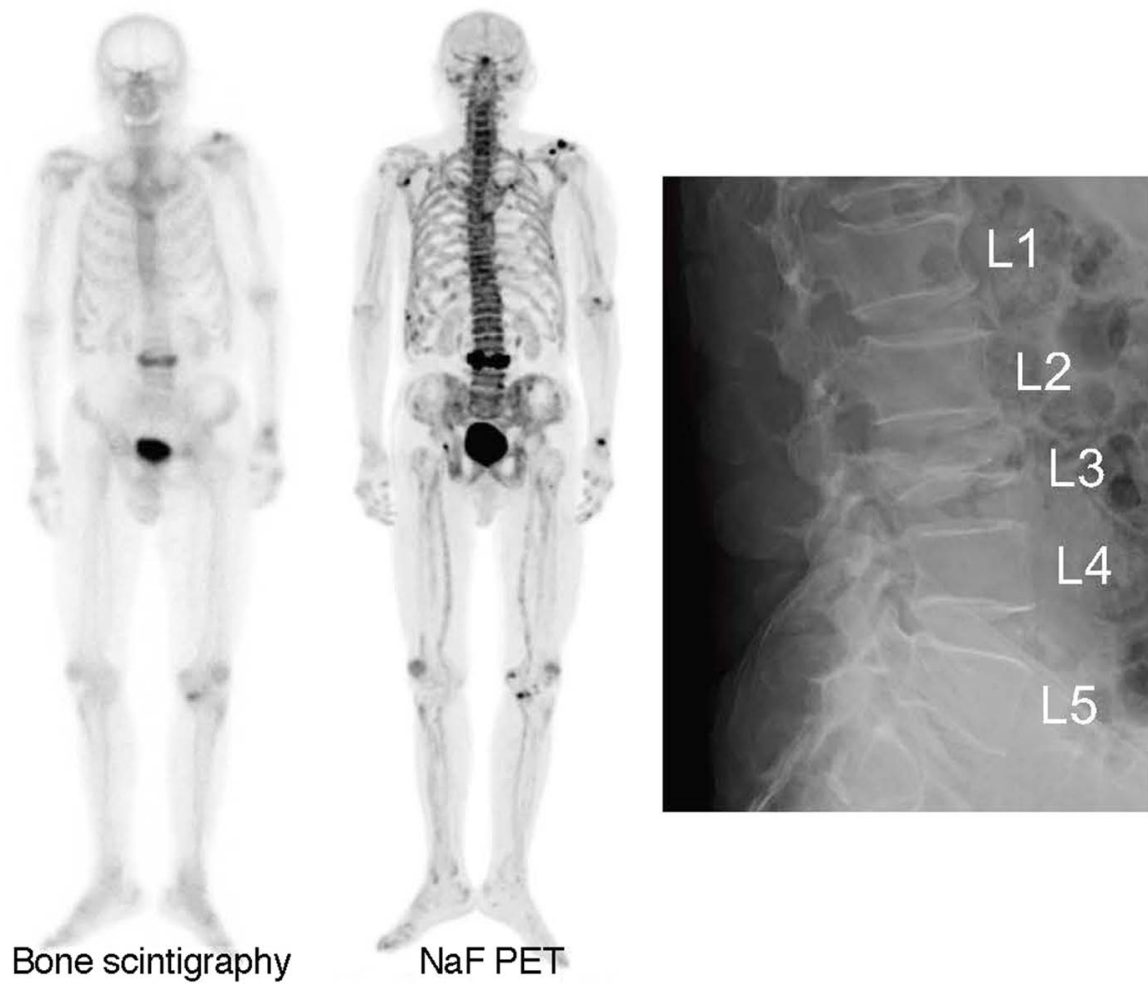

Figure S1. A patient with an L3 osteoporotic compression fracture. Bone scintigraphy (left panel) and NaF PET (right panel) images in the anteroposterior view were obtained in the same week. In addition to the L3 vertebra, tracer accumulation in the left shoulder and the left wrist was clearly observed on NaF PET, suggestive of degenerative joint disease. These lesions are not apparent on bone scintigraphy. 